\title{
Validity and reliability of the motivation for physical activity (RM4-FM) questionnaire
}

\author{
Mikko Uimonen ${ }^{1, *}$, Jussi P. Repo', Kiira Grönroos ${ }^{2}$, Arja Häkkinen ${ }^{2,3}$, Simon Walker ${ }^{4}$ \\ 'Department of Surgery, Central Finland Health Care District, Jyväskylä, Finland \\ ${ }^{2}$ Health Sciences, Faculty of Sport and Health Sciences, University of Jyäskylä, Jyväskylä, Finland \\ ${ }^{3}$ Department of Physical Medicine, Central Finland Health Care District, Jyväskylä, Finland \\ ${ }^{4}$ NeuroMuscular Research Center, Faculty of Sport and Health Sciences, University of Jyväskylä, Finland
}

There is a lack of validated instruments measuring motivation for physical activity (RM4-FM) in the Finnish language. The study examined the translated RM4-FM instrument's psychometric properties in a sample of healthy, older Finnish adults. RM4-FM was translated and linguistically validated adhering to published guidelines. $65-75$-year olds $(n=102)$, completed the RM4-FM, the physical activity acceptance questionnaire (PAAO) and sociodemographic characteristics questionnaires electronically. The RM4-FM was readministered one month after the initial assessment. RM4-FM translated well into Finnish. Motivation dimension scores were skewed towards high internal and low external motivation. A floor effect was confirmed in the external regulation dimension and a ceiling effect was confirmed in the identified regulation dimension. Confirmatory factor analysis indicated inadequacy of the four-dimension model. Separate assessment of each of the four motivation dimensions with Exploratory factor analysis showed unidimensionality for all dimen- sions. Exploratory factor analysis provided a best-fit model of three factors (influence of other people, intention of well-being and emotional aspect of motivation). Readministration of the instruments showed good test-retest reliability in all motivation dimensions. Female gender, higher education, and higher PAAC score were associated with higher internal motivation and lower external motivation scores. The Finnish version of the RM4-FM instrument provides valid and reliable scores in assessing motivation for physical activity in healthy, older Finnish adults. The score distributions suggested shortages in the scale when evaluating internal motivation. The three-factor model of the RM4-FM provided more favorable structural validity compared to the original version.

Keywords: Self-determination theory, Inactivity, Psychometric properties, Patient-reported outcome measure

\section{INTRODUCTION}

Physical activity has a key role promoting well-being and quality of life (Bize et al., 2007). Inactivity has been shown to be a major risk factor for several chronic diseases, such as cardiovascular diseases, diabetes mellitus type 2, osteoporosis and colon cancer as well as psychological disorders (Penedo and Dahn, 2005). The prevalence of such diseases in Western countries is high (Chen et al., 2011; Haggar and Boushey 2009; Hernlund et al., 2013; Kessler and Bromet 2013; Sanchis-Gomar et al., 2016; Townsend et al., 2016). In addition, cardiovascular diseases are a major cause of death (Sanchis-Gomar et al., 2016; Townsend et al., 2016). Over- all, the burden of such diseases for society are substantial measured in costs and healthy years lived (Vos et al., 2015). The evidence of the positive effects of physical activity on the risk and, on the other hand, the prognosis of the chronic diseases is indisputable (Penedo and Dahn, 2005). Despite these positive effects on health, inactivity is still a major issue in public health (Blair, 2009; Kohl et al., 2012).

On an individual level, a key point of the decision of whether to be physically active is motivation. The self-determination theory by Ryan and Deci (2000) divides motivation into controlled and autonomous aspects. In the field of physical activity, the autonomous aspect of motivation refers to the intention of joy, well-be-
${ }^{*}$ Corresponding author: Mikko Uimonen (D https://orcid.org/0000-0001-6609-1345 Department of Surgery, Central Finland Health Care District, Keskussairaalantie 19, 40620 Jyväskylä, Finland

Email: mikko.uimonen@tuni.fi

Received: March 2, 2021 / Accepted: March 25, 2021
This is an Open Access article distributed under the terms of the Creative Commons Attribution Non-Commercial License (https://creativecommons.org/licenses/by-nc/4.0/) which permits unrestricted non-commercial use, distribution, and reproduction in any medium, provided the original work is properly cited. 
ing and achievement of personal goals derived from physical activity, while the controlled aspect refers to the influence and the pressure of others, as well as the guilt of not exercising (Ryan and Deci, 2000). Self-determination theory proposes the importance of the motivational factors originating from oneself, rather than external factors, on the determination between physically active or physically inactive lifestyles. Strong intrinsic motivation has been shown to be associated with positive long-term effects of physical activity, as intrinsically motivated individuals continue being active even when the influence of external pressure has stopped (Ryan and Deci, 2000; Teixeira et al., 2012). Thus, to achieve permanent positive change in one's physical activity habits, it is crucial to enhance the intrinsic motivation aspect.

Lately, the development of interventions on strengthening the motivation for physical activity has been a subject of interest in the field of sport science and medicine (Silva et al., 2010; Vanroy et al., 2019). Such interventions have been effective in enhancing internal motivation for physical activity (e.g., Silva et al., 2010). However, it is important to identify the individuals who are at risk for physical inactivity-related diseases and have issues with their motivation to provide targeted interventions to those who benefit most. For this purpose, several questionnaires have been developed (Deci, 2004; Murcia et al., 2007).

The motivation for physical activity (RM4-FM) questionnaire is a self-administered instrument developed to assess the internal and external aspects of physical activity motivation (Deci, 2004). RM4-FM was developed based on self-determination theory. RM4-FM has been utilized to examine motivation for physical activity (Nurmi et al., 2016), yet it has not been properly validated for this purpose. In addition, modified versions of RM4-FM have been used in assessing motivation for dieting and quitting smoking (Levesque et al., 2007).

Validation and understanding of psychometric properties of measurement instruments is indispensable for correct use of these questionnaires (Mokkink et al., 2010a, 2010b). Proper validation processes enable researchers to evaluate the usability of the instrument when assessing whether to choose the instrument when initiating a new study. Additionally, proper validation creates the frames for interpreting and assessing the meaning of the results obtained from these instruments.

Hence, the psychometric properties of the RM4-FM questionnaire have not been examined properly. In addition, the instrument has not been translated or validated for the Finnish population. The aim of the present study was to translate the RM4-FM instrument into Finnish and examine the psychometric properties of the translated version in a sample of healthy, older adults.

\section{MATERIALS AND METHODS}

The study was conducted as part of an exercise intervention study examining the effect of resistance training frequency on strength, functional capacity and health outcomes (NCT02413112). The study was approved by the Ethics Committee of the University of Jyväskylä (2309/2013), adhered to the Declaration of Helsinki (2013), and all subjects provided informed consent. The study contacted two-thousand 65-75-year old people living in the Jyväskylä city region by recruitment mail, who were identified based on age and selected randomly from the Population Register. Those who were willing to participate to the study $(\mathrm{n}=454)$ completed an electronic preliminary registration questionnaire that was used to select individuals to an information meeting $(\mathrm{n}=148)$. The individuals with health issues that may hamper involvement to physical activity (notable overweight with body mass index (BMI) $37 \mathrm{~kg} / \mathrm{m}^{2}$ or above, poorly controlled cardiovascular disease, or lower extremity injury that may complicate physical training and testing, a need of assistive device, use of medication that influences the neurological or endocrinal functions) or who already involved regularly in physical exercise or sport (over 180 min per week) or had other factors which may affect the physical performance and exercise (previous experience of strength training, previous testosterone treatment, smoking) were excluded. After all, 102 suitable individuals signed informed consent to participate and were included to the study. The baseline characteristics of the participants are shown in Table 1. The participants completed the RM4-FM and the physical activity acceptance questionnaire (PAAQ) questionnaires electronically, as well as a questionnaire on sociodemographic characteristics. The participants were asked to recomplete the RM4-FM questionnaire one month after the initial assessment.

\section{RM4-FM: motivation for physical activity}

RM4-FM is a 16-item questionnaire measuring the four dimensions of motivation for physical activity (Deci, 2004). All items use a 7-point Likert-scale. The RM4-FM is divided in four dimensions of four items: external regulation, introjected regulation, identified regulation, and intrinsic motivation. The score of each dimension is calculated as a mean of the scores of that dimension's items. Higher scores indicate stronger influence of motivation within the specific dimension. In addition, the relative autonomy index (RAI) is calculated by using a formula including all four motivation dimension scores ( $\mathrm{RAI}=2 \times$ intrinsic motivation + identified regulation-intro- 
Table 1. Sociodemographic and clinical characteristics of the participants $(\mathrm{n}=102)$

\begin{tabular}{lc}
\hline Characteristic & Value \\
\hline Women & $57(56)$ \\
Age $(\mathrm{yr})$ & $69 \pm 3$ \\
Body mass index $\left(\mathrm{kg} / \mathrm{m}^{2}\right)$ & $28 \pm 4$ \\
Physical activity per week $(\mathrm{min})$ & $104 \pm 61$ \\
Education & \\
Basic education or general upper secondary & $65(64)$ \\
University or college & $37(36)$ \\
Marital status & \\
Married or living with the spouse & $79(77)$ \\
Divorced, widow or unmarried & $23(23)$ \\
Chronic diseases & \\
Cardiovascular & $32(31)$ \\
Respiratory & $10(10)$ \\
Musculoskeletal & $11(11)$ \\
Diabetes & $7(7)$ \\
Gastrointestinal & $3(3)$ \\
Neurological & $3(3)$ \\
Other & $17(17)$ \\
Using medication & $52(51)$ \\
Physical activity acceptance questionnaire score & $50 \pm 8$ \\
\hline
\end{tabular}

Values are presented as number (\%) or mean \pm standard deviation.

jected regulation $-2 \times$ external regulation). $\mathrm{RAI}$ indicates the relative impact of intrinsic and extrinsic factors in motivation for physical activity. A negative RAI value indicates that extrinsic factors have stronger influence on motivation while a positive RAI value indicates stronger influence of intrinsic factors on motivation, and thus autonomy.

\section{Physical activity acceptance questionnaire}

The PAAQ is a 10-item questionnaire measuring the subjective psychological and physical discomfort caused by physical activity (Butryn et al., 2015). All items use a 7-point Likert-scale and the maximum score is 70 . Lower scores indicate higher amount of discomfort caused by physical activity and problems with motivation to continue such activity. The PAAQ has been shown to be valid and reliable in measuring psychological factors behind motivation for physical activity (Butryn et al., 2015).

\section{Translation and cultural adaptation process}

The translation process and linguistic validation of the RM4-FM questionnaire adhered to the guidelines of Wild et al. (2005). Two native Finnish academic researchers of sport science independently forward-translated the RM4-FM into Finnish language. These two translated versions were then compared and reconciliated. The reconciliated version was then back-translated to English by a native English-speaking researcher that is also fluent in Finnish. After back-translation, discrepancies between forward- and backtranslated versions of RM4-FM were corrected and accordance between the original and translated versions was verified. Finally, all researchers that participated in the translation process proofread and approved the final version.

\section{Statistical analysis}

The sociodemographic and clinical data are presented as means, standard deviations (SDs) and 95\% confidence intervals (CIs) or as counts with percentages. Normality of the RM4-FM and its dimensions score distributions were assessed. Floor and ceiling effects were examined from baseline measurement to assess the scale targeting. If $15 \%$ of patients scored minimum or maximum points, the floor or ceiling effect was considered confirmed (Mokkink, 2010a).

To test cross-cultural validity, the differences in the motivation dimension scores between gender, educational and relationship status subgroups were assessed using independent samples $t$-test. Pearson correlation coefficients were calculated to assess the association between RM4-FM and sociodemographic characteristics of the participants. Furthermore, convergence with the PAAQ score was examined by calculating Pearson correlation coefficients for each dimension as well as for RAI against the PAAQ score. The coefficient values of $0.3,0.5,0.7$, and 0.9 were interpreted as low, moderate, strong and very strong correlation, respectively (Mukaka, 2012).

To assess the structural validity of RM4-FM, confirmatory factor analysis (CFA) was conducted. The presumed factor structure consisting of four motivation dimensions was tested. The chi-square test of model fit, the root mean square error of approximation (RMSEA), the comparative fit index (CFI), and the standardized root mean square residual (SRMR) were calculated. A nonsignificant chi-square test value, RMSEA less than 0.05 , CFI over 0.95 and SRMR less than 0.80 were interpreted as good fit of the tested model (Prinsen et al., 2018). The unidimensionality of the RM4FM dimensions were examined with exploratory factor analysis (EFA). In addition, a best-fit factor model was explored with EFA. Varimax rotation with the Kaiser normalization method was used to obtain the most distinguishable factor structure. A factor loading of 0.4 was used as a cutoff value.

Internal consistency of RM4-FM and its dimensions were assessed using Cronbach alpha with bootstrapping method of 1,000 
repetitions to obtain the $95 \% \mathrm{CIs}$. Alpha values over 0.7 were considered as acceptable (Prinsen et al., 2018). Intraclass correlation coefficients (ICCs) and 95\% CIs were calculated to assess testretest-reliability. ICC over 0.7 was interpreted as sufficient stability, while ICC values under 0.7 represent unacceptable stability (Prinsen et al., 2018; Terwee et al., 2007).

The statistical analyses were conducted with R and IBM SPSS Statistics ver. 25.0 (IBM Co., Armonk, NY, USA). Interpretation and reporting of the results of this study adhere to the COnsensusbased Standards for the selection of health status Measurement INstruments (COSMIN) guidelines (Prinsen et al., 2018). The present study was conducted as part of a larger study investigating the effects of resistance training on functional capacity (Turpela et al., 2017), health (Ihalainen et al., 2019), and psychological wellbeing (Kekäläinen et al., 2018). The study protocol was approved by The Ethical Committee of The University of Jyväskylä.

\section{RESULTS}

\section{Translation}

RM4-FM translated well into Finnish. The comparison of the two forward-translated versions of RM4-FM revealed minor linguistic differences that did not change the content of the questionnaire. In addition, the cross-checking of back-translated and the original version of the RM4-FM showed no substantial discrepancies. After minor adjustments, the translated version of RM4-FM was approved by the researchers (Supplementary material 1).

\section{Sample characteristics}

A total of 102 eligible participants completed the questionnaires in their entirety. Table 1 presents the sociodemographic and clinical characteristics of the participants. The mean body mass index (BMI) of the participants was 28 indicating mild overweight. While a proportion of participants undertook medication for blood pressure, cholesterol and/or blood glucose, none had serious injury or illness and could be considered healthy and cognitively proficient for this age group. Variation in the average duration of physical activity per week was high ranging from 0 to $200 \mathrm{~min}$.

\section{Distribution of the RM4-FM}

Fig. 1 presents the distributions of each RM4-FM dimensions. Distribution of external regulation score was skewed towards low scores while distributions of identified regulation and intrinsic motivation scores were skewed towards high scores. Introjected regulation score and RAI were normally distributed. The coverage of the dis- tribution was good in introjected regulation dimension while in other dimensions the skewness of the distribution impaired the coverage. A floor effect was confirmed in the external regulation dimension and a ceiling effect was confirmed in the identified regulation dimension (Table 2). No other floor or ceiling effects were observed.

\section{Cross-cultural validity}

The examination of differences in motivation dimension scores and RAI between subgroups by gender revealed, that external regulation score was higher among male (2.6 vs. $2.1, P=0.030)$ and mean RAI of female participants was higher than in males ( 9.8 vs. $7.8, P=0.009)$. No other significant differences were observed between genders. The external regulation (1.9 vs. 2.6, $P=0.003)$ and introjected regulation (3.7 vs. $4.3, P=0.027$ ) scores of participants with higher education were lower compared to basic educated participants. Other dimension scores or RAI did not differ between educational subgroups. In addition, marital status was not associated to dimension scores or RAI.

The Pearson correlation coefficients of age, BMI and physical activity time with RM4-FM dimensions and RAI showed no notable correlations. Yet, the correlations of BMI with introjected regulation score $(r=-0.273, P=0.006)$ and intrinsic motivation score $(r=-0.20, P=0.045)$, and of physical activity time with intrinsic motivation score $(r=0.276, P=0.006)$, identified regulation score $(r=0.21, P=0.034)$ and introjected regulation score $(r=0.20, P=$ $0.048)$ were statistically significant but still of negligible strength. PAAQ scores correlated positively to RAI $(r=0.422, P<0.001)$, intrinsic motivation $(r=0.394, P<0.001)$ and identified regulation $(r=0.341, P<0.001)$ scores. Nevertheless, the strength of the correlations was low.

\section{Structural validity}

The results of CFA indicated poor fit of the tested four-dimension factor model of RM4-FM, as the chi-square test of model fit $\left(\chi^{2}=256, d f=98, P<0.001\right)$, the RMSEA value 0.126 (90\% CI, $0.107-0.145 ; P<0.001$ ), the CFI value 0.811 and the SRMR 0.103 demonstrated inadequacy of the model. On the other hand, when unidimensionality of each RM4-FM dimension was tested separately with EFA, all motivation dimensions showed unidimensionality as only one factor with Eigenvalue over 1 was found for each dimension. Furthermore, all items in each dimension loaded strongly (loading value over 0.4 ) on these identified factors. Internal consistency of the RM4-FM was good as the Cronbach alpha was 0.88 (95\% CI, 0.83-0.91). In addition, the alphas of the RM4-FM di- 

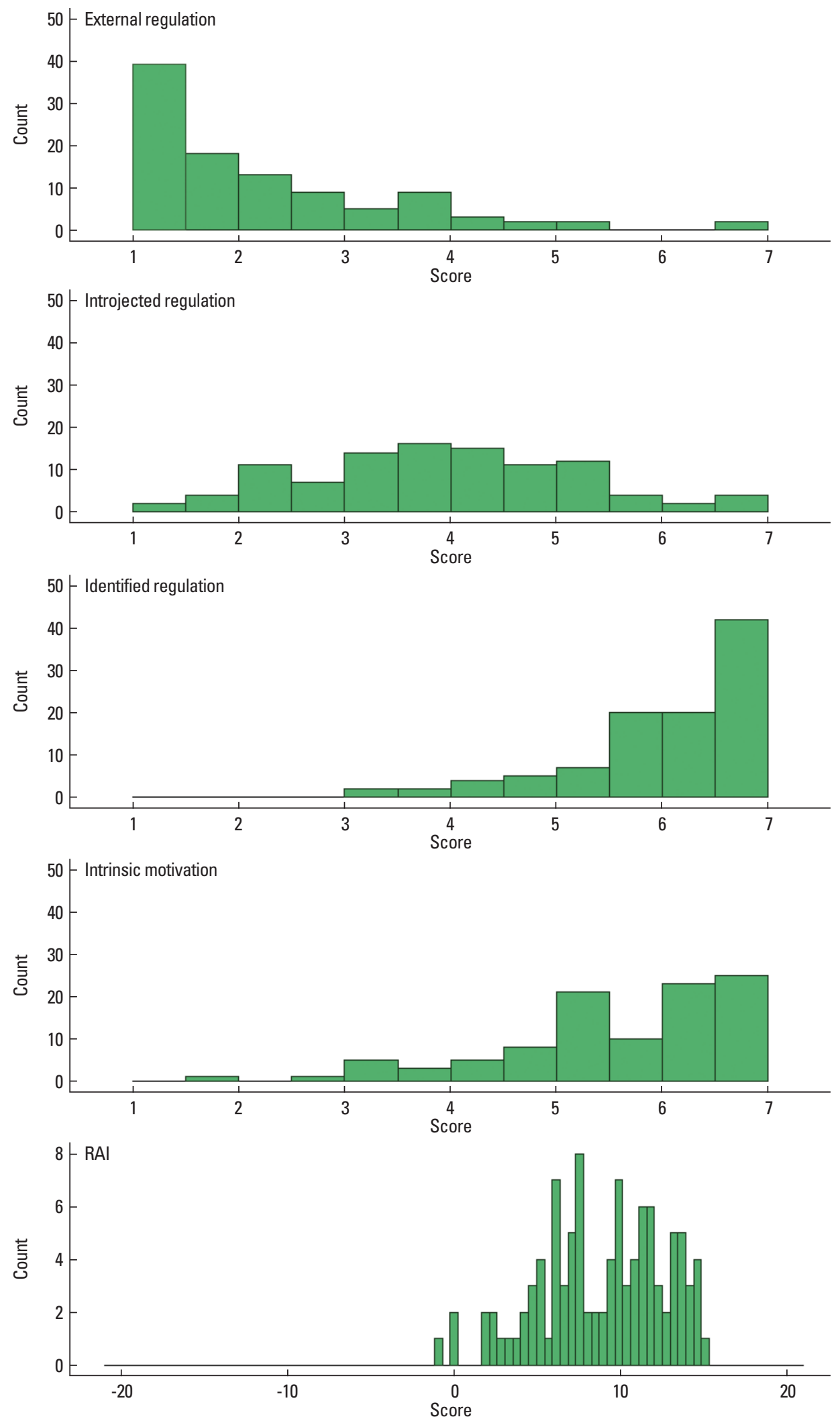

Fig. 1. Distributions of individual motivation for physical activity (RM4-FM) questionnaire dimension scores and relative autonomy index (RAI). 
Table 2. Mean scores, Cronbach alphas, and percentage of maximum and minimum points of motivation for physical activity (RM4-FM) questionnaire dimensions

\begin{tabular}{lcccc}
\hline Dimension & Mean \pm SD & $\begin{array}{c}\text { Alpha } \\
(95 \% \mathrm{Cl})\end{array}$ & $\begin{array}{c}\text { Minimum } \\
(\%)\end{array}$ & $\begin{array}{c}\text { Maximum } \\
(\%)\end{array}$ \\
\hline External regulation & $2.3 \pm 1.3$ & $0.82(0.72-0.89)$ & 21.6 & 2.0 \\
Introjected regulation & $4.1 \pm 1.3$ & $0.75(0.63-0.83)$ & 0 & 2.9 \\
Identified regulation & $6.2 \pm 0.9$ & $0.72(0.60-0.80)$ & 0 & 21.6 \\
Intrinsic motivation & $5.7 \pm 1.1$ & $0.84(0.76-0.90)$ & 0 & 11.8 \\
\hline
\end{tabular}

$\mathrm{SD}$, standard deviation; $\mathrm{Cl}$, confidence interval.

Table 3. Factor loadings of the items in the best-fit factor model for the motivation for physical activity (RM4-FM) questionnaire

\begin{tabular}{clccc}
\hline Item No. & \multicolumn{1}{c}{ Dimension } & Factor 1 & Factor 2 & Factor 3 \\
\hline 1 & Introjected regulation & 0.23 & 0.27 & 0.54 \\
2 & External regulation & 0.80 & -0.03 & 0.17 \\
3 & Intrinsic motivation & -0.02 & 0.31 & 0.79 \\
4 & Introjected regulation & 0.50 & 0.16 & 0.60 \\
5 & Identified regulation & 0.06 & 0.25 & 0.71 \\
6 & Introjected regulation & 0.79 & 0.01 & 0.20 \\
7 & External regulation & 0.83 & 0.02 & 0.05 \\
8 & Intrinsic motivation & 0.23 & 0.61 & 0.47 \\
9 & Identified regulation & -0.10 & 0.67 & 0.40 \\
10 & Intrinsic motivation & -0.02 & 0.52 & 0.64 \\
11 & External regulation & 0.82 & -0.05 & 0.19 \\
12 & Identified regulation & 0.21 & 0.79 & 0.18 \\
13 & Introjected regulation & 0.36 & 0.17 & 0.52 \\
14 & External regulation & 0.73 & 0.32 & -0.18 \\
15 & Intrinsic motivation & 0.06 & 0.81 & 0.25 \\
16 & Identified regulation & -0.06 & 0.76 & 0.24 \\
\hline
\end{tabular}

mensions showed acceptable internal consistency with values over 0.7 (range, 0.72-0.84) (Table 2).

Examination of the best-fit factor model of RM4-FM with EFA revealed three factors with eigenvalues over 1 (Table 3). Factor 1 (Eigenvalue $=6.0$ ) consisted of all items of external regulation and one item of introjected regulation dimensions, which all emphasize the influence of other people on motivation (items 2, 6, 7, 11, and 14). Factor 2 (Eigenvalue $=3.1$ ) consisted of two items of intrinsic motivation and three items of identified regulation dimensions (items $8,9,12,15$, and 16). The items of factor 2 concentrate on intention of well-being. Three items of introjected regulation, two items of intrinsic motivation and one item of identified regulation dimensions formed factor 3 (Eigenvalue $=1.1$, items 1, 3, 4, 5, 10, and 13). Factor 3 incorporates the items on emotional aspect of motivation comprising the items on joy of physical activity and, on the other hand, guilt of inactivity. Cronbach alphas of these factors

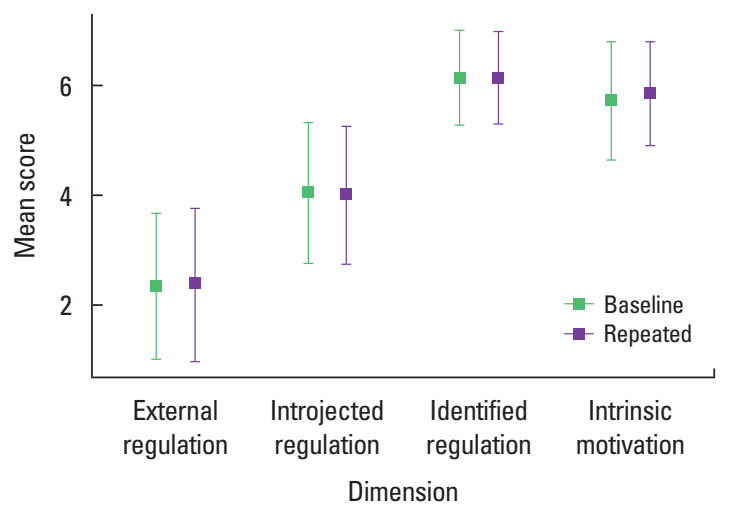

Fig. 2. Comparison of baseline and repeated (RM4-FM) dimension scores. RM4-FM, motivation for physical activity.

were $0.86,0.85$, and 0.81 for factors 1,2 , and 3 , respectively.

\section{Test-retest reliability}

Eighty-one participants (79\%) recompleted the RM4-FM. Fig. 2 shows the baseline and repeated measure scores of RM4FM dimensions. The ICC values of RM4-FM dimensions of intrinsic motivation (ICC $=0.87 ; 95 \% \mathrm{CI}, 0.79-0.92$ ), external regulation (ICC $=0.85 ; 95 \% \mathrm{CI}, 0.76-0.90)$ and identified regulation (ICC $=0.80 ; 95 \%$ CI, 0.68-0.87) indicated good stability while ICC value of introjected regulation (ICC $=0.78$; 95\% CI, 0.66-0.86) showed moderate stability. Mean RAI remained stable in baseline and repeated measures (8.9 in baseline vs. 9.2 in repeated). The ICC of RAI (ICC $=0.86$; 95\% CI, 0.78-0.91) indicated good stability.

\section{DISCUSSION}

The main finding of this study was that the Finnish version of RM4-FM provides valid and reliable scores in physical activity motivation assessment. Nevertheless, our findings showed limitations regarding the targeting of the dimension scores and a need for consideration of factor structure of RM4-FM (Table 4).

There is a lack of validated instruments concerned with motivation of physical activity for the Finnish population. In this study, the translation and cross-cultural validation of the RM4-FM was performed according to internationally submitted guidelines (Wild et al., 2005). There were only minor discrepancies between two forward-translated versions, as well as between the original and backward translated versions, and these discrepancies did not affect the content of the instrument. Furthermore, the examination of the association of sociodemographic factors and RM4-FM scores 
Table 4. Predefined hypotheses and conclusions for validation of the motivation for physical activity (RM4-FM) questionnaire

\begin{tabular}{|c|c|c|}
\hline Feature & Hypothesis & Conclusion \\
\hline \multicolumn{3}{|l|}{ Coverage and targeting } \\
\hline \multirow[t]{10}{*}{ Floor and ceiling effect } & No floor effect (min score $<15 \%$ ) & \\
\hline & Intrinsic motivation & Confirmed \\
\hline & Identified regulation & Confirmed \\
\hline & Introjected regulation & Confirmed \\
\hline & External regulation & Rejected \\
\hline & No ceiling effect (max score <15\%) & \\
\hline & Intrinsic motivation & Confirmed \\
\hline & Identified regulation & Rejected \\
\hline & Introjected regulation & Confirmed \\
\hline & External regulation & Confirmed \\
\hline \multicolumn{3}{|l|}{ Validity } \\
\hline Cross-cultural validity & $\begin{array}{l}\text { Nonsignificant associations between } \\
\text { the RM4-FM score and } \\
\text { sociodemographic characteristics }\end{array}$ & $\begin{array}{l}\text { Confirmed/ } \\
\text { rejected }\end{array}$ \\
\hline $\begin{array}{l}\text { Convergence with the } \\
\text { PAAO }\end{array}$ & $\begin{array}{l}\text { Significant and at least low correlation } \\
\text { between the PAAQ and the RM4-FM } \\
\text { scores }\end{array}$ & $\begin{array}{l}\text { Confirmed/ } \\
\text { rejected }\end{array}$ \\
\hline \multicolumn{3}{|l|}{ Structural validity } \\
\hline \multirow[t]{4}{*}{ CFA } & Chi-square test nonsignificant & Rejected \\
\hline & RMSEA $<0.05$ & Rejected \\
\hline & $\mathrm{CFI}>0.95$ & Rejected \\
\hline & SRMR $<0.80$ & Rejected \\
\hline \multirow[t]{5}{*}{ EFA } & Unidimensional structure of dimensions & \\
\hline & Intrinsic motivation & Confirmed \\
\hline & Identified regulation & Confirmed \\
\hline & Introjected regulation & Confirmed \\
\hline & External regulation & Confirmed \\
\hline \multirow[t]{5}{*}{ Internal consistency } & Cronbach alpha is $>0.7$ & \\
\hline & Intrinsic motivation & Confirmed \\
\hline & Identified regulation & Confirmed \\
\hline & Introjected regulation & Confirmed \\
\hline & External regulation & Confirmed \\
\hline \multicolumn{3}{|l|}{ Reliability } \\
\hline \multirow[t]{5}{*}{ Test-retest reliability } & ICC $>0.7$ & \\
\hline & Intrinsic motivation & Confirmed \\
\hline & Identified regulation & Confirmed \\
\hline & Introjected regulation & Confirmed \\
\hline & External regulation & Confirmed \\
\hline
\end{tabular}

CFA, confirmatory factor analysis; EFA, exploratory factor analysis; RMSEA, root mean square error of approximation; $\mathrm{CF}$, comparative fit index; SRMR, standardized root mean square residual; ICC, intraclass correlation coefficient; PAAQ, physical activity acceptance questionnaire.

indicated good cross-cultural validity of RM4-FM.

The scale targeting of RM4-FM dimensions was not optimal on the basis of the distributions and coverage of each dimension scores.
The scores of the dimensions associated to internal motivation (intrinsic motivation and identified regulation) were skewed towards high scores and a ceiling effect was confirmed in identified regulation dimension, whereas external regulation score was skewed towards low scores and was confirmed with a floor effect. The result suggests that applicability of RM4-FM is limited in a sample of highly internally motivated people. A study by Nurmi et al. (2016) investigated the mediator effect of self-regulation in the association between intrinsic motivation and participation in physical activity in adolescents using a broadly similar questionnaire with minor differences (SRQ-E). The scores of intrinsic motivation and identified regulation dimensions in that study were focused around high scores with mean scores 3.84 (SD, 0.97) and 3.85 (SD, 0.96), respectively. On the other hand, the mean scores of external regulation and introjected regulation were 1.78 (SD, 0.85) and 2.78 (SD, 1.09). The distributions of the scores in the study by Nurmi et al. (2016) and in the present study were very similar supporting the suggestion that scale targeting and ceiling effects might be a problem independent of age group.

The CFA showed that RM4-FM did not obey the presumed four-dimension structure. The results of CFA on the four-dimensional model indicated weak structural validity of RM4-FM. In addition, despite the sufficiency of Cronbach alphas of the RM4FM and its dimensions, the values were relatively low, especially within the individual dimensions. Low Cronbach alphas of the dimensions may be due to a low number (4) of items in each dimension, but, on the other hand, it may reflect that items within the dimensions do not measure the same construct. The EFA produced a best-fit model of three factors: influence of other people, intention of well-being and emotions of physical activity. The three-factor model showed at least comparable, if not better, performance in psychometric properties compared to the original model. However, despite the strengths of the three-factor model, it is limited due to losing the basis and division in motivation dimensions based on the self-determination theory. The three-factor model would provide specific information on motivation from a different perspective than the original model. Nevertheless, the EFA of single RM4-FM dimensions showed unidimensionality of each dimension, which in turn support the sufficiency of structural validity and applicability of the four-dimensional model of RM4-FM.

High ICC values indicated high test-retest reliability with 1 -month recompletion interval. Motivation for physical activity is a dynamic phenomenon that may vary depending on changing situations and mood of one's life. High ICC suggests that RM4FM is not strongly affected by these varying circumstances, but it 
rather reflects more permanent aspects of motivation.

There were some limitations in the present study. Firstly, criterion validity of RM4-FM was not measured, as there was not a sufficient instrument to compare the results. Secondly, the study sample was limited to older individuals (i.e., 65-75 years of age), which, in addition, may be influenced by a high rate of chronic diseases and medication use in the sample. The age and health status of the participants may have also affected habits and attitudes, as well as motivation for physical activity, particularly since the present sample were highly functionable for their age despite the fact that they were not physically activity. The advantages of this study were a large randomly selected sample from the Population Register, which represents well the average population as the regularly physically active individuals were excluded, as well as comprehensive translation and statistical methods, which adhere to the international guidelines on questionnaire validation studies. In addition, the questionnaires were completed electronically, and the questionnaire program required the participant to complete all the items, thus, preventing missing data.

In conclusion, the Finnish version of RM4-FM instrument provides valid and reliable scores in assessing motivation for physical activity in a sample of healthy, older Finnish adults. However, regarding the floor and ceiling effects of the dimensions, the scale seemed to be improperly targeted suggesting shortages in the scale when evaluating internally motivated subjects. A three-factor model of the RM4-FM provided more favorable psychometric properties than the original version though it loses the basis of self-determination theory. The three-factor model is a noteworthy alternative when assessing motivation for physical activity using the RM4-FM.

\section{CONFLICT OF INTEREST}

No potential conflict of interest relevant to this article was reported.

\section{ACKNOWLEDGMENTS}

The authors received no financial support for this article.

\section{SUPPLEMENTARY MATERIAL}

Supplementary material 1 can be found via https://doi.org/10. 12965/jer.2142194.097.

\section{REFERENCES}

Bize R, Johnson JA, Plotnikoff RC. Physical activity level and health-related quality of life in the general adult population: a systematic review. Pre Med 2007;45:401-415.

Blair SN. Physical inactivity: the biggest public health problem of the 21st century. Br J Sports Med 2009;43:1-2.

Butryn ML, Arigo D, Raggio GA, Kaufman AI, Kerrigan SG, Forman EM. Measuring the ability to tolerate activity-related discomfort: initial validation of the physical activity acceptance questionnaire (PAAQ). J Phys Act Health 2015;12:717-726.

Chen L, Magliano DJ, Zimmet PZ. The worldwide epidemiology of type 2 diabetes mellitus - present and future perspectives. Nat Rev Endocrinol 2011;8:228-236.

Deci EL. Exercise self-regulation questionnaires. Self-determination theory: an approach to human motivation and personality - the self-regulation questionnaires. 2004 [cited 2020 Nov 9]. Available from: www. selfdeterminationtheory.org/self-regulation-questionnaires.

Haggar FA, Boushey RP. Colorectal cancer epidemiology: incidence, mortality, survival, and risk factors. Clin Colon Rectal Surg 2009;22:191197.

Hernlund E, Svedbom A, Ivergård M, Compston J, Cooper C, Stenmark J, McCloskey EV, Jönsson B, Kanis JA. Osteoporosis in the european enion: medical management, epidemiology and economic burden. A report prepared in collaboration with the international osteoporosis foundation (IOF) and the european federation of pharmaceutical industry associations (EFPIA). Arch Osteoporos 2013;8:136.

Thalainen JK, Inglis AJ, Mäkinen T, Newton RU, Kainulainen H, Kyröläinen $\mathrm{H}$, Walker S. Strength training improves metabolic health markers in older individual regardless of training frequency. Front Physiol 2019;10:32.

Kekäläinen T, Kokko K, Tammelin T, Sipila S, Walker S. Motivational characteristics and resistance training in older adults: a randomized controlled trial and 1-year follow-up. Scand J Med Sci Sports 2018;28: 2416-2426.

Kessler RC, Bromet EJ. The epidemiology of depression across cultures. Annu Rev Public Health 2013;34:119-138.

Kohl HW 3rd, Craig CL, Lambert EV, Inoue S, Alkandari JR, Leetongin G, Kahlmeier S, Lancet Physical Activity Series Working Group. The pandemic of physical inactivity: global action for public health. Lancet 2012;380:294-305.

Levesque CS, Williams GC, Elliot D, Pickering MA, Bodenhamer B, Finley PJ. Validating the theoretical structure of the treatment self-regulation questionnaire (TSRQ) across three different health behaviors. Health Educ Res 2007;22:691-702. 
Mokkink LB, Terwee CB, Knol DL, Stratford PW, Alonso J, Patrick DL, Bouter LM, de Vet HCW. The COSMIN checklist for evaluating the methodological quality of studies on measurement properties: a clarification of its content. BMC Med Res Methodol 2010a;10:22.

Mokkink LB, Terwee CB, Patrick DL, Alonso J, Stratford PW, Knol DL, Bouter LM, de Vet HCW. The COSMIN checklist for assessing the methodological quality of studies on measurement properties of health status measurement instruments: an international Delphi study. Qual Life Res 2010b;19:539-549.

Mukaka MM. Statistics corner: a guide to appropriate use of correlation coefficient in medical research. Malawi Med J 2012;24:69-71.

Murcia J, Gimeno EC, Camacho AM. Measuring self-determination motivation in a physical fitness setting: validation of the behavioural regulation in exercise questionnaire-2 (BREQ-2) in a Spanish sample. J Sports Med Phys Fitness 2007;47:366-378.

Nurmi J, Hagger MS, Haukkala A, Araújo-Soares V, Hankonen N. Relations between autonomous motivation and leisure-time physical activity participation: the mediating role of self-regulation techniques. J Sport Exerc Psychol 2016;38:128-137.

Penedo FJ, Dahn JR. Exercise and well-being: a review of mental and physical health benefits associated with physical activity. Curr Opin Psychiatry 2005;18:189-193.

Prinsen CA, Mokkink LB, Bouter LM, Alonso J, Patrick DL, de Vet HCW, Terwee CB. COSMIN guideline for systematic reviews of patient-reported outcome measures. Qual Life Res 2018;27:1147-1157.

Ryan RM, Deci EL. Self-determination theory and the facilitation of intrinsic motivation, social development, and well-being. Am Psychol 2000; 55:68-78.

Sanchis-Gomar F, Perez-Quilis C, Leischik R, Lucia A. Epidemiology of coronary heart disease and acute coronary syndrome. Ann Transl Med 2016;4:256.
Silva MN, Vieira PN, Coutinho SR, Minderico CS, Matos MG, Sardinha LB, Teixeira PJ. Using self-determination theory to promote physical activity and weight control: a randomized controlled trial in women. J Behav Med 2010;33:110-122.

Teixeira PJ, Silva MN, Mata J, Palmeira AL, Markland D. Motivation, selfdetermination, and long-term weight control. Int J Behav Nutr Phys Act 2012;9:22.

Terwee CB, Bot SD, de Boer MR, van der Windt AWM, Knol DL, Dekker J, Bouter LM, de Vet HCW. Quality criteria were proposed for measurement properties of health status questionnaires. J Clin Epidemiol 2007; 60:34-42.

Townsend N, Wilson L, Bhatnagar P, Wickramasinghe K, Rayner M, Nichols M. Cardiovascular disease in Europe: epidemiological update 2016. Eur Heart J 2016;37:3232-3245.

Turpela M, Häkkinen K, Haff GG, Walker S. Effects of different strength training frequencies on maximum strength, body composition and functional capacity in healthy older individuals. Exp Gerontol 2017; 98:13-21.

Vanroy, J, Seghers J, van Uffelen J, Boen F. Can a framed intervention motivate older adults in assisted living facilities to exercise? BMC Geriatr 2019;19:46.

Vos T, Barber RM, Bell B, Global Burden of Disease Study 2013 Collaborators. Global, regional, and national incidence, prevalence, and years lived with disability for 301 acute and chronic diseases and injuries in 188 countries, 1990-2013: a systematic analysis for the global burden of disease study 2013. Lancet 2015;386:743-800.

Wild D, Grove A, Martin M. Principles of good practice for the translation and cultural adaptation process for patient-reported outcomes (PRO) measures: report of the ISPOR task force for translation and cultural adaptation. Value Health 2005;8:94-104. 